is very important to astrophysics, and it does not yet enter heavily in the laboratory experiment of aerodynamicists. When we introduce a radiation field into the equation of aerodynamics, we get into trouble with the equations because they become incredibly complicated. We have tried once or twice a linearized approach to the motions, including the radiation field and even then things are horribly complicated.

\title{
Closing remarks to the meeting by M. Minnaert.
}

I should like to present a few general impressions from this interesting meeting of scientists working in two different fields. It has often been noticed that progress in science is made just on such meeting points, and I think this specially applies to astronomy in combination with other sciences. Astronomy in the last 50 years passed successively through the age of optics, then of atomic physics, then of nuclear physics, and now again of aerodynamics and electro-magnetism. At first sight I get the impression that the contribution of aerodynamics to the development of astrophysics has been less spectacular, less sudden perhaps than the progress made at the moment when Bohr's atomic theory was discovered and applied by SAHA to stellar atmospheres; or at the moment when principles of nuclear physics were discovered, and fusion processes were found to be the main source of energy in cosmical bodies and the source of evolution. But this might well be a perspective effect. In the time when you are living, you always have the impression that things are going slowly; and when you are looking back, then you see that really in that period in a short time a considerable progress was achieved.

Such concepts as convection, shock-waves, magnetohydrodynamic waves are so fundamental now in astrophysics that we could not miss them. They are part of our vocabulary, they play such an important role that looking backwards, perhaps in 10 or 20 years, we may also have the impression that this was really considerable progress in a short time.

It is clear a priori that the contribution of aerodynamics to astronomy must be very important; for after all, with very few exceptions, our universe is composed of gases. It is an aero-universe, and more specifically it is not. only an aero-universe but an aero-dynamic universe. Everywhere we find that there are motions in stellar and interstellar space, even in these layers of the sun and the stars where you would have thought that there was at least radiative equilibrium. We find that inside that same layer there is "microturbulence "; and in deeper layers there are motions of convection; and then there are the chromosphere and the corona with their shock-waves. Everywhere there are motions; the whole universe is really an aerodynamic universe. It is clear by adding to astronomy the knowledge which we can get from aero- 
dynamical science, a considerable number of new possibilities arise for astrophysicists, of which we have explored only a small part-we are only just starting.

To aerodynamicists it must certainly be interesting to see how their work can be applied to cosmical problems. There are quite a number of new questions which have been put by astronomy and which must be stimulating in all directions for aerodynamicists. However, it will be necessary that astronomers try to put their problems in a simplified and clear form. The construction of good models is one of the important and difficult tasks of science, one of the greatest mean of progress. I think that we should also train our students in making models, and that we generally don't; we give them models, and they have to calculate what are the consequences. Let them learn to transform the complications of nature into a simple model, which afterwards may be treated by well-known mathematical methods. If we compare the application of aerodynamics to the study of interstellar space, and its application to the study of stellar atmospheres, we see that there is this important difference, that the application to interstellar space could hardly be tested by laboratory experiments. There, we are confronted with immense spaces and extremely low densities, which could not be imitated in any way, and the only way to progress is just theoretical calculation and argument. But in the case of stellar atmospheres, there is a great promise in the possibility of making laboratory experiments; this came out very clearly in many aspects during this colloquium and must incite aerodynamicists to giving their attention to the experimental solution of the problems with which we are concerned here. In the meeting of scientists of two different groups, there is always a certain difficulty to be overcome before one understands the language of the other one. One might hope that between aerodynamics and astrophysics, there would be a common language which they both understood-that is mathematics. But it appears that this does not really help so much, because the difficulties of common understanding are not in the mathematical process, but in the translation from the language of aerodynamics into mathematics or in the translation of astronomy into mathematics. It is there that the difficulties are. Once you have made the model and the translation, the mathematical machine operates and there we all understand each other.

It seems to me that in certain respects we have over-estimated each other's knowledge. We could have perhaps prepared the audience better for the general lectures. Astronomers should have shown more slides about solar ṕhenomena and should have made things a little more picturesque. In a similar. way there are basic concepts of aerodynamics which were given often after they had already been used extensively in the introductions. These are things which occur quite naturally and would have been difficult to avoid. But for future meetings let us try to introduce always the necessary preliminary con- 
cepts, let us encourage people to ask for explanations, to look very clearly at the fundamentals and not to be satisfied with general terminology but to ask critically about the basic points.

It may look to astronomers that some of the aerodynamical theories are somewhat far from astrophysical applications. On the other hand it is clear that we must first give our full attention to such pieces of theory, which are, so to say, machines which can be applied to many different cases. We must understand how a certain number of these machines work to be able to put them together afterwards, and to construct a whole factory, giving the results which we need in order to explain our observations. From that point of view, astronomers should have the patience to listen, to understand, to become familiar with different parts of aerodynamical machinery, even if they do not see at once the possibility of application.

It is a disturbing circumstance that often in the discussion it becomes clear that the astronomical observations, on which we should be glad to rely, are not so certain as one would have hoped; and that on the other side, the aerodynamical theories, with which the astronomers were at first so happy, are not so well-established either. And that on both sides there are doubts and contradictions, and people thinking in another way. I can give no consolation in this respect except to say that fortunately the complaints are reciprocal. But I think we have to be resigned-this is the difficulty of science on the march, that there are inevitably a certain number of doubtful points which must be overcome by gradual progress.

It seems to me that after what we have heard, we shall feel the necessity of reading more of each other's work; and in that respect I should like from the point of view of astronomers to ask that in the reports of this symposium there should be given a certain number of good references to general aerodynamical books. Secondly, and perhaps more important, that also specified references should be mentioned by those having quoted papers of the literature in the course of their introductions or in the discussions.

I think that on both sides, we had sometimes the impression that the other group puts too much emphasis on details, which might seem irrelevant. There has been a discussion on the temperature of the corona, and $I$ heard an aerodynamicist saying "Well, is it so important whether that is one or two million degrees?" One must understand that in a certain number of cases such an apparent detail is very important, because it may disclose fundamental questions concerning the structure of such a stellar layer. And also from the other side, astronomers sometimes had similar impressions. The mixing-length theory, for example, which gives very useful and practical results and is extensively used, was criticized by aerodynamicists because it is not quite accurate in all details. It is clear that it is not easy to say where precision becomes essential and where it is simply a nicety which does not matter. This has to 
be left to the critical sense and appraisal of each group, and no general rules can be given. It is only clear that, where details are not essential and only a matter of discussion between specialists, they should be left out in colloquia like this.

I have heard the question being put: "Will it be in the future necessary for students of astronomy to take a course in aerodynamics? " I would be inclined to say: Yes, certainly, and I think in many universities our students are having already such a course. Unfortunately, such lectures always give too much on one side and too little on the other side. It is impossible to have a special course of aerodynamics made in such a fashion that it precisely applied to the needs of astronomers. That would not be right, even-they have to learn something about aerodynamical science in broad lines, and then they must be able for themselves to study more especially these parts which are important for astronomy. One may hope that sooner or later there will be written a textbook on aerodynamical astronomy or something like that-especially applying to astronomers, just as we have now already textbooks of cosmical electro-magnetism or about radio astronomy, especially written for the astronomical applications. But let me ask also the reciprocal question: "Will it in the future be necessary for students in aerodynamics to take a course in astronomy?" There I am afraid that I cannot say yes. But I hope that aerodynamicists shall insert in their lectures a certain number of examples taken from astronomy, which may be very impressive because always it is beautiful to see how theoretical physics can be applied to nature on a cosmical scale.

I am afraid that I have perhaps emphasized a little too much the difficulties of common understanding-on the other side there is no doubt that for the great part, we have been able to overcome these difficulties, and that very fine and interesting results have been reached in this colloquium. They have been explained and summarized by several people here. I only want to emphasize particularly the very beautiful observational results which have been presented and which are fully appreciated when we take in mind the immense amount of work of organization, of perseverance which is necessary before such data are obtained. We must also remember that a quantity of new information may be derived out of this material. On the other hand, it is gratifying that there has been not only a series of beautiful observational results and a series of aerodynamical theories-but that on many points the connection has been made; for that was the essential moment of this colloquium and its real purpose. 